\title{
Some geometric properties of the Witten genus
}

\author{
Anand Dessai \\ Abstract. We give a survey on geometric properties of the Witten genus. \\ The survey focuses on relations between the Witten genus, group actions and \\ positive curvature.
}

\section{Introduction}

A striking feature of spin geometry is the rich interplay between curvature, symmetries and index theory. To illustrate this we recall two classical results. Firstly, Lichnerowicz proved that the Dirac operator on a closed spin manifold $M$ of positive scalar curvature is invertible. In particular, its index, the $\hat{A}$-genus $\hat{A}(M)$, vanishes. Secondly, Atiyah and Hirzebruch applied the Lefschetz fixed point formula to the equivariant Dirac operator to show that the $\hat{A}$-genus vanishes on a connected closed spin manifold with smooth non-trivial action by the circle.

According to Witten the free loop space $\mathcal{L} M$ of a manifold $M$ should carry an $S^{1}$-equivariant "Dirac operator" if $w_{1}(M)=w_{2}(M)=0$ (i.e. if $M$ admits a spin structure) and $\frac{p_{1}}{2}(M)=0$. Here the $S^{1}$-action on $\mathcal{L} M$ is given by reparametrization of loops. It is well-known that these topological constraints are equivalent to the existence of a lift of the classifying map of the stable tangent bundle $M \rightarrow B O$ to $B O\langle 8\rangle$, the 7 -connected cover of $B O$. Following modern terminology we shall call such a lift a string structure and $M$ a string manifold.

Unfortunately, a mathematically rigorous construction of a Dirac operator on free loop spaces is still missing. However, by formally applying the Lefschetz fixed point formula to the equivariant index of the hypothetical "Dirac operator" on a string manifold $M$ and using zeta-function renormalization Witten derived an honest topological invariant of $M$, the Witten genus $\varphi_{W}(M)$. It turns out that many geometric properties of the $\hat{A}$-genus on spin manifolds have counterparts for the Witten genus on string manifolds.

The purpose of this paper is to survey some of these analogues and to point towards some open questions/problems which arise naturally in this context. This

2000 Mathematics Subject Classification. Primary 58J26, Secondary 53C20, 57S15, 57R20.

Key words and phrases. Witten genus, group actions, positive curvature.

Part of the research was supported by the SFB 478 Münster, the DFG-Schwerpunkt 1154 and SNF Grant No. 200021-117701. 
survey is very much inspired by the paper [36] of Stephan Stolz in which he explains his conjecture about the vanishing of the Witten genus on string manifolds of positive Ricci curvature.

Our exposition is rather incomplete. It focuses on group actions, the conjecture of Stolz and its connections to the Witten genus and other rational genera. In particular, we neither discuss homotopy theoretical refinements of the Witten genus and the construction of elliptic cohomology via topological modular forms by Hopkins and Miller [22] nor the work of Stolz and Teichner on elliptic objects [37].

In the next section we recall the $\hat{A}$-vanishing theorem of Atiyah and Hirzebruch and discuss relations between the Witten genus and group actions on string manifolds. In Theorem 2.8 we show that the kernel of the Witten genus can be represented rationally by string manifolds with non-abelian symmetry. This result relies on the construction of a suitable basis sequence of Cayley plane bundles (see Proposition 2.9) which is carried out in Section 5. In Section 3 we discuss basic instances in which Stolz' conjecture is known to hold. We also look at the rational bordism invariants which obstruct the existence of a metric of positive Ricci curvature on all spin manifolds. In dimension $\leq 100$ we show that the $\hat{A}$-genus is essentially the only such invariant (see Proposition 3.5). In Section 4 we look at the problem of describing geometrically the universal multiplicative genus for Cayley plane bundles. This section is more speculative.

Some of the results presented in this survey are new (see Proposition 3.4, Proposition 3.5) or have only been published in preprint form (see Theorem 2.8, Proposition 2.9 ). It is my pleasure to thank the referee for many valuable suggestions.

\section{Symmetries}

A classical problem is to understand symmetries of a given closed manifold. For continuous symmetries (by which we mean: smooth actions of compact connected Lie groups) on spin manifolds this problem is closely related to the Dirac operator.

Let $G$ be a compact Lie group acting smoothly on a spin manifold $M$. After averaging a Riemannian metric and passing to a two-fold covering action, if necessary, we may assume that $G$ acts isometrically on $M$ preserving the spin structure. In this situation a $G$-equivariant Dirac operator can be constructed. Its index $\hat{A}(M)_{G}$ is an element of the complex representation $\operatorname{ring} R(G)$. We identify $\hat{A}(M)_{G}$ with a function on $G$ via its character.

Atiyah and Hirzebruch applied the Lefschetz fixed point formula [2] to the equivariant Dirac operator to prove the

THEOREM 2.1 ( $\hat{A}$-vanishing theorem [1]). Let $M$ be a connected spin manifold with smooth non-trivial action by a compact Lie group $G$. If $G$ is connected then the equivariant $\hat{A}$-genus $\hat{A}(M)_{G}$ vanishes identically.

It is easy to see that the conclusion fails in general if $M$ is not spin or $G$ is not connected. Moreover, Burghelea has constructed examples of topological $S^{1}$-actions on smooth spin manifolds with non-vanishing $\hat{A}$-genus (cf. [9, p. 184]).

In general, any rigidity statement for a genus can be rephrased in terms of multiplicativity properties in certain fibre bundles (cf. [32, Prop. 1]). In particular, the $\hat{A}$-vanishing theorem is equivalent to the following 
THEOREM 2.2. The Â-genus vanishes on any smooth fibre bundle of closed oriented manifolds provided that the fibre is a spin manifold and the structure group is a compact connected Lie group which acts smoothly and non-trivially on the fibre.

The $\hat{A}$-genus of an $n$-dimensional spin manifold refines to a $K O$-theoretical invariant, the $\alpha$-invariant $\alpha(M) \in K O_{n}(p t)$, which captures additional $\mathbb{Z} / 2 \mathbb{Z}$ information in dimension $n \equiv 1,2 \bmod 8$. The question of how this refinement is related to continuous symmetries is quite subtle. On the one hand one obtains spin manifolds with free $S^{1}$-action and non-vanishing $\alpha$-invariant by taking the product of $S^{1}$ (equipped with the interesting spin structure) with any $8 k$-dimensional spin manifolds of odd $\hat{A}$-genus [28] (simply connected examples are discussed for example in $[\mathbf{2 5}$, p. 232]). On the other hand Lawson and Yau showed that any manifold with non-trivial $S^{3}$-action admits a metric of positive scalar curvature [25]. Since the $\alpha$-invariant vanishes on spin manifolds of positive scalar curvature $[\mathbf{2 1}]$ it follows that the $\alpha$-invariant is an obstruction to $S^{3}$-actions on spin manifolds. It would be interesting to give a purely topological proof of this fact which does not rely on the construction of a metric of positive scalar curvature.

Atiyah and Hirzebruch observed that rationally the converse of the $\hat{A}$-vanishing theorem also holds. Recall that the rational oriented bordism ring and the rational spin bordism ring are isomorphic, $\Omega_{*}^{S O} \otimes \mathbb{Q} \cong \Omega_{*}^{S p i n} \otimes \mathbb{Q}$. It is well-known that a $K_{3}$-surface and the quaternionic projective spaces $\mathbb{H} P^{k}, k \geq 2$, constitute a rational basis sequence of $\Omega_{*}^{S O}$, i.e. $\Omega_{*}^{S O} \otimes \mathbb{Q} \cong \mathbb{Q}\left[K_{3}, \mathbb{H} P^{2}, \mathbb{H} P^{3}, \ldots\right]$. The $K_{3}$-surface, being a complex surface with holonomy $S U(2)$, has $\hat{A}\left(K_{3}\right)=2$. The $\hat{A}$-genus vanishes on the quaternionic projective spaces (for example by Theorem 2.1). Hence, the rational bordism class of any manifold with vanishing $\hat{A}$-genus belongs to the ideal in $\Omega_{*}^{S O} \otimes \mathbb{Q} \cong \Omega_{*}^{S p i n} \otimes \mathbb{Q}$ generated by the quaternionic projective spaces. This gives

THEOREM $2.3([\mathbf{1}])$. Let $M$ be a spin manifold $M$ with $\hat{A}(M)=0$. Then a non-zero multiple of $M$ is spin cobordant to a spin manifold $M^{\prime}$ which admits a non-trivial smooth $S^{1}$-action on each of its components.

It is not difficult to construct a rational basis sequence $\left\{M_{4 k}\right\}_{k \geq 1}$ of the oriented bordism ring such that $M_{4 k}$ is a $K_{3}$-surface for $k=1$ and an $\mathbb{H} P^{2}$-bundle with structure group $S^{1}$ and non-trivial $S^{3}$-action along the fibres for $k \geq 2$ (the proof follows the strategy of the proof of Proposition 2.9). Hence, any spin manifold $M$ of dimension $\geq 8$ with $\hat{A}(M)=0$ is rationally cobordant to an $\mathbb{H} P^{2}$-bundle with non-trivial $S^{3}$-action along the fibres.

Kreck and Stolz showed the much deeper result that any spin manifold $M$ of dimension $\geq 8$ with $\alpha(M)=0$ is integrally cobordant to the total space of an $\mathbb{H} P^{2}$-bundle with structure group $P S p(3)$ (cf. [23, Prop. 3.5]). Since $P S p(3)$ is the full isometry group of $\mathbb{H} P^{2}$ with its standard metric it is not clear (at least to the author) whether their construction can be modified to yield examples with continuous symmetries. If possible, it would immediately imply a positive solution to the following

QUESTION 2.4. Let $M$ be a spin manifold $M$ with $\alpha(M)=0$. Is $M$ spin cobordant to a spin manifold which admits a non-trivial smooth $S^{1}$-action on each of its components? 
We shall now discuss relations between the Witten genus and group actions. For an introduction to the Witten genus we refer to [40] and [20, Section 6.3] and [36]. The Witten genus $\varphi_{W}(M)$ of a $4 k$-dimensional spin manifold $M$ is defined by the following series of indices of twisted Dirac operators

$$
\begin{gathered}
\varphi_{W}(M)=\hat{A}\left(M ; \bigotimes_{n=1}^{\infty} S_{q^{n}} T M\right) \cdot \prod_{n=1}^{\infty}\left(1-q^{n}\right)^{4 k}= \\
\left(\hat{A}(M)+\hat{A}(M ; T M) \cdot q+\hat{A}\left(M ; T M+S^{2}(T M)\right) \cdot q^{2}+\ldots\right) \cdot \prod_{n=1}^{\infty}\left(1-q^{n}\right)^{4 k} \in \mathbb{Z}[[q]] .
\end{gathered}
$$

Here $T M$ is the tangent bundle of $M, q$ is a formal variable, $S_{t}=\sum S^{i} \cdot t^{i}$ denotes the symmetric power operation and $\hat{A}(M ; E)$ is the index of the Dirac operator twisted with the complexified vector bundle $E \otimes \mathbb{C}$.

Note that the definition of the Witten genus can be extended to oriented manifolds using the cohomological version of the index theorem. This yields the genus $\varphi_{W}: \Omega_{*}^{S O} \rightarrow \mathbb{Q}[[q]]$ which corresponds to the power series $x / \Phi(x)$, where $\Phi(x)$ denotes the Weierstrass' $\Phi$-function (for string-manifolds one may replace $\Phi(x)$ by the Weierstrass' $\sigma$-function).

If the first Pontrjagin class of $M$ vanishes rationally (or more generally: if all Pontrjagin numbers involving $p_{1}(M)$ vanish) then $\varphi_{W}(M)$ is the Fourier expansion of a modular form for $S L_{2}(\mathbb{Z})$ of weight $2 k$.

Now assume $M$ is a string manifold. By the discussion above $\varphi_{W}(M)$ is a modular form for $S L_{2}(\mathbb{Z})$ with integer coefficients. Hence, the Witten genus defines a ring homomorphism from the string bordism ring $\Omega_{*}^{\text {string }}$ to the ring of integral modular forms for $S L_{2}(\mathbb{Z})$.

According to Witten $[\mathbf{4 0}] \varphi_{W}(M)$ is best thought of as the $S^{1}$-equivariant index of a hypothetical "Dirac operator" on the free loop space $\mathcal{L} M$ of $M$. In the presence of an action by a Lie group $G$ one would ideally like to compute the index of the $G$-equivariant "Dirac operator" by localizing using a Lefschetz fixed point type formula. So far, neither the operator has been constructed nor such formula has been proved. However, a mathematical rigorous analysis can be carried out for the equivariant Witten genus $\varphi_{W}(M)_{G}$ which one obtains by replacing the indices in the power series for the Witten genus by their equivariant refinements.

Suppose $M$ is a string manifold with non-trivial smooth action by $G=S^{1}$. Liu proved that the equivariant Witten genus $\varphi_{W}(M)_{S^{1}} \in R\left(S^{1}\right)[[q]]$ vanishes identically provided that the first equivariant Pontrjagin class of $M$ lies in the image of the map $H_{S^{1}}^{4}(p t ; \mathbb{Z}) \rightarrow H_{S^{1}}^{4}(M ; \mathbb{Z})$ induced from the projection $M \rightarrow p t$ (cf. [27, Th. 4, p. 370]). If the second Betti number of $M$ vanishes this is easily seen to be the case. As observed in [10] Liu's condition on the equivariant first Pontrjagin class is also fulfilled if the $S^{1}$-action (or a suitable covering action) extends to an action by a semi-simple Lie group (in the case of homogeneous spaces this was independently shown by Gerald Höhn). This gives the following

THEOREM $2.5(\hat{A}$-vanishing theorem for $\mathcal{L} M[\mathbf{2 7}, \mathbf{1 0}])$. Let $M$ be a connected string manifold with smooth non-trivial action by a compact connected Lie group $G$. If $G$ is semi-simple then the equivariant Witten genus $\varphi_{W}(M)_{G} \in R(G)[[q]]$ vanishes identically. In particular, the Witten genus vanishes on connected string manifolds with non-trivial smooth $S^{3}$-action. 
In terms of multiplicativity properties in fibre bundles the vanishing theorem may be rephrased as

THEOREM 2.6. The Witten genus vanishes on any smooth fibre bundle of closed oriented manifolds provided the fibre is a string manifold and the structure group is a compact connected semi-simple Lie group which acts smoothly and non-trivially on the fibre.

For a proof of this vanishing theorem and various generalizations we refer to $[\mathbf{2 7}, \mathbf{1 1}]$. As remarked above the conclusion of the theorem also holds for $S^{1}$-actions provided the second Betti number of $M$ vanishes. However, the following question appears to be still unresolved.

Question 2.7. Does the Witten genus vanish on connected string manifolds with smooth non-trivial $S^{1}$-action?

Rationally the vanishing of the Witten genus for $S^{3}$-actions given in Theorem 2.5 has the following converse.

THEOREM 2.8. Let $M$ be a string manifold with $\varphi_{W}(M)=0$. Then a non-zero multiple of $M$ is string cobordant to a string manifold which is the total space of a Cayley plane bundle with structure group $S^{1}$ and non-trivial $S^{3}$-action along the fibres. In particular, $M$ is rationally string cobordant to a string manifold which admits a non-trivial smooth $S^{3}$-action on each of its components.

The theorem is a consequence of the following construction which might be of independent interest.

Proposition 2.9. The oriented bordism ring has a rational basis sequence $\left\{M_{4 k}\right\}_{k \geq 1}$, i.e. $\Omega_{*}^{S O} \otimes \mathbb{Q} \cong \mathbb{Q}\left[M_{4}, M_{8}, \ldots\right]$, such that

(1) $M_{4 k}$ is a connected $4 k$-dimensional spin manifold for all $k$,

(2) $M_{4 k}$ is a string manifold for $k \geq 2$,

(3) $M_{4 k}$ is the total space of a Cayley plane bundle with structure group $S^{1}$ and smooth non-trivial $S^{3}$-action along the fibres for $k \geq 4$.

The two results above were proved in my unpublished preprint [10] (see Section 5 for the argument).

\section{Positive curvature}

By the work of Lichnerowicz [26] and Hitchin [21] the $\hat{A}$-genus and its $K O$ theoretical refinement, the $\alpha$-invariant, vanish on a closed spin manifold of positive scalar curvature. Conversely, one knows from Stolz' solution [35] of the GromovLawson conjecture that any simply connected spin manifold $M$ of dimension $\geq 5$ admits a metric of positive scalar curvature provided the $\alpha$-invariant $\alpha(M)$ vanishes.

In [36] Stolz argued that a metric of positive Ricci curvature on $M$ should be related to "positive scalar curvature" on the free loop space $\mathcal{L} M$. This reasoning led Stolz to conjecture the following loop space analogue of Lichnerowicz' theorem.

ConjeCture 3.1 (Stolz' conjecture [36]). Let $M$ be a string manifold. If $M$ admits a metric of positive Ricci curvature then the Witten genus $\varphi_{W}(M)$ vanishes.

Any spin manifold of positive Ricci curvature has positive scalar curvature and, hence, vanishing $\hat{A}$-genus. This fact alone already implies the conjecture in 
dimension $4 k<24$ or dimension 28. In dimension 24 or dimension $4 k \geq 32$, however, Stolz' conjecture is open even if one restricts to manifolds of positive sectional curvature. A proof of the conjecture in any of these dimensions would provide first examples of simply connected Riemannian manifolds of positive scalar curvature which do not admit a metric of positive Ricci curvature (cf. [36, 6.1]). This underlines the geometric significance of the conjecture.

We shall now discuss some evidence for the conjecture in two basic instances. Further evidence can be obtained using fibre bundle constructions (see [36, Th. $3.1])$.

A basic source for positive Ricci curvature is Kähler geometry. By Yau's solution [41] of the Calabi conjecture any Kähler manifold with positive first Chern class carries a metric of positive Ricci curvature. This prompts the following question which in our opinion is one of the central questions in the context of Stolz' conjecture.

Question 3.2. Does the Witten genus vanish on string Kähler manifolds with positive first Chern class?

Among these manifolds Stolz' conjecture is known to hold for certain subfamilies. For example Landweber and Stong (cf. [20, p. 87]) have shown that the Witten genus vanishes on those complete intersections in complex projective space which are string (it is straightforward to check that these manifolds have positive first Chern class). Recall that complex projective spaces are irreducible Hermitian symmetric. The question whether Stolz' conjecture holds for string manifolds which are complete intersections in irreducible Hermitian symmetric spaces other than complex projective spaces was considered by Förster in his $\mathrm{PhD}$ thesis [16]. Among other things Förster was able to prove the vanishing of the Witten genus for string complete intersections in the exceptional symmetric spaces $E_{6} / \operatorname{Spin}(10) \times U(1)$ and $E_{7} / E_{6} \times U(1)$.

Next to Kähler geometry compact Lie groups provide the main other source for positive Ricci curvature. It is well-known (see for example [3]) that a compact homogeneous space admits an invariant metric of positive Ricci curvature if its fundamental group $\pi_{1}$ is finite (recall that the condition on $\pi_{1}$ is necessary by Myers' theorem). In [17] Grove and Ziller showed that invariant metrics of positive Ricci curvature also exist on manifolds of cohomogeneity one with finite $\pi_{1}$. Subsequently, Schwachhöfer and Tuschmann exhibited metrics of positive Ricci curvature on manifolds with $\pi_{1}$ finite which are quotients of homogeneous spaces or quotients of spaces of cohomogeneity one [33]. More recently, Böhm and Wilking [4] proved that any manifold of non-negative sectional curvature and $\pi_{1}$ finite admits a metric of positive Ricci curvature.

It is easy to see that any homogeneous manifold with finite fundamental group and any manifold of cohomogeneity one with finite fundamental group admits an almost effective action by $S^{3}$. Hence, Theorem 2.5 gives the following

Corollary 3.3. Stolz' conjecture is true for homogeneous manifolds and manifolds of cohomogeneity one.

If one wants to extend this argument to quotients one faces the problem that quotients may be quite unsymmetric. To illustrate this consider a biquotient $G / / H$ of a compact Lie group $G$, i.e. $G / / H$ is the quotient of $G$ by a closed subgroup $H \subset G \times G$ which acts freely on $G$ with respect to the two-sided action of $G \times G$ on 
$G$. The obvious symmetries of $G / / H$ are given by the normalizer $N:=\operatorname{Norm}_{G \times G}(H)$ which may not allow for non-trivial homomorphisms $S^{3} \rightarrow N$.

In [14] we consider the conjecture of Stolz for biquotients of a simple Lie group $G$. Using Theorem 2.6 the conjecture can be reduced to the situation where $H$ is maximal of maximal rank in $G$. We then apply Eschenburg's classification (cf. [15, Kapitel 10]) of these subgroups to show that for each string biquotient in the classification the Witten genus vanishes. Unfortunately, as we learned from Jost Eschenburg, his classification is incomplete and one still needs to check Stolz' conjecture in the cases not covered in [15].

In view of the result of Böhm and Wilking [4] Stolz' conjecture predicts the vanishing of the Witten genus for simply connected string manifolds of non-negative sectional curvature. As pointed out before the conjecture is not known for this class of manifolds even if one restricts to the subclass of manifolds of positive sectional curvature. In this connection we would like to mention the following consequence of the vanishing theorems for spin manifolds of positive sectional curvature and symmetry given in $[\mathbf{1 2}, \mathbf{1 3}]$. Recall that the symmetry rank of a Riemannian manifold is the rank of its isometry group.

Proposition 3.4. Let $M$ be a string manifold of dimension $4 k \leq 44$ or $4 k=$ 52. Suppose $M$ admits a metric of positive sectional curvature with symmetry rank $\geq 2$. Then the Witten genus of $M$ vanishes.

Proof. Recall that the Witten genus of a $4 k$-dimensional string manifold $M$ is the Fourier expansion of a modular form for $S L_{2}(\mathbb{Z})$ of weight $2 k$. The first two coefficients are given (up to a constant) by the index of the Dirac operator and the index of the Dirac operator twisted with the tangent bundle, i.e.

$$
\varphi_{W}(M)=(\hat{A}(M)+\hat{A}(M, T M) \cdot q+\ldots) \cdot \prod_{n=1}^{\infty}\left(1-q^{n}\right)^{4 k} .
$$

Recall also that the ring $M_{*}\left(S L_{2}(\mathbb{Z})\right)$ of modular forms for $S L_{2}(\mathbb{Z})$ is a polynomial ring generated by the Eisenstein series $E_{4}=1+240 \cdot q+2160 \cdot q^{2}+\ldots$ and $E_{6}=1-504 \cdot q-16632 \cdot q^{2}+\ldots$ which are of weight 4 and 6 , respectively.

By $[\mathbf{2 6}, \mathbf{1}]$ the $\hat{A}$-genus of a spin manifold vanishes if the manifolds admits a metric of positive scalar curvature or a non-trivial torus action. Hence, $\varphi_{W}(M)$ is a cusp form. Since there are no non-trivial cusp forms of weight $<12$ the proposition follows in dimension $<24$. In $[\mathbf{1 3}]$ we showed that in dimension $>8$ the index $\hat{A}(M, T M)$ vanishes for spin manifolds of positive sectional curvature with symmetry rank $\geq 2$. Hence, $\varphi_{W}(M)$ is a modular form which has a zero of order $\geq 2$ at infinity. It follows from the expansions of the Eisenstein series above and the structure of $M_{*}\left(S L_{2}(\mathbb{Z})\right)$ that non-trivial modular forms with a zero of order 2 at infinity occur only in weight 24 and weight $\geq 28$. This completes the proof of the proposition.

It follows from [12] that the conclusion also holds if a two dimensional torus acts effectively and smoothly on $M$ and the torus contains two involutions acting isometrically on $M$. Note however that the vanishing of the Witten genus may well be a consequence of the existence of a smooth effective $S^{1}$-action alone (see Question 2.7 above).

We close this section with a discussion of rational bordism invariants (i.e. nontrivial linear combinations of Pontrjagin numbers) which obstruct the existence of 
a metric of positive Ricci curvature. We first observe that for oriented manifolds there aren't any since the rational oriented bordism ring is generated by complex projective spaces and these have positive sectional curvature with respect to their standard metric. For spin manifolds Lichnerowicz' result tells us that the $\hat{A}$-genus obstructs positive scalar curvature and, hence, positive Ricci curvature as well. For string manifolds the conjecture of Stolz predicts additional obstructions encoded in the Witten genus.

Somewhat related to Stolz' conjecture is the problem of determining in a given dimension those rational bordism invariants which vanish for all spin manifolds of positive Ricci curvature. In particular, one wants to know whether the $\hat{A}$-genus is (up to multiples) the only rational bordism invariant which vanishes on these manifolds. A positive answer would imply that a spin manifold of positive scalar curvature is (after passing to a suitable multiple) spin bordant to a (not necessarily connected) manifold of positive Ricci curvature. A negative answer would yield examples of simply connected manifolds of positive scalar curvature which do not admit a metric of positive Ricci curvature.

We believe that the $\hat{A}$-genus is essentially the only rational bordism invariant which obstructs positive Ricci curvature on spin manifolds. This is supported by the following

Proposition 3.5. In dimension $n \leq 100$ any rational bordism invariant which vanishes on all spin manifolds of positive Ricci curvature is a multiple of the $\hat{A}$ genus.

IDEA OF PROOF. The idea is to find in any given dimension $n=4 k$ spin manifolds of positive Ricci curvature which generate a linear subspace $V_{4 k}$ of $\Omega_{4 k}^{s p i n} \otimes \mathbb{Q}$ of codimension one. Once this is accomplished the assertion follows, since the $\hat{A}$ genus induces an isomorphism $\Omega_{4 k}^{s p i n} \otimes \mathbb{Q} / V_{4 k} \cong \mathbb{Q}$. Unfortunately, it is not known whether the elements in the rational spin bordism ring which can be represented by manifolds of positive Ricci curvature form an ideal. This accounts for the difficulties involved in the construction of the subspace $V_{4 k}$.

In dimension $n=4 k \leq 100$ we can show that the set of spin complete intersections with positive first Chern class generate a subspace $V_{4 k}$ of $\Omega_{4 k}^{\text {spin }} \otimes \mathbb{Q}$ of codimension one. By Yau's solution [41] of the Calabi-conjecture $V_{4 k}$ is generated by spin manifolds of positive Ricci curvature. The computations make heavy use of computer power which is the reason for the present constraint on the dimension. We expect that a more intelligent way of dealing with the linear algebra involved will show the proposition in all dimensions.

\section{Multiplicativity in Cayley plane bundles}

This section is about (rational) genera which are multiplicative in Cayley plane bundles. Prominent examples are the Witten genus and the elliptic genus (the "signature" of the free loop space). A challenging problem is to give a geometric description of the universal multiplicative genus for Cayley plane bundles.

We begin with a discussion of multiplicative genera for homogeneous vector bundles in general. Let $G$ be a compact connected Lie group and $H$ a connected closed subgroup. By definition, a $G / H$-bundle is a smooth fibre bundle $E \rightarrow B$ of oriented closed manifolds with fibre $G / H$ and structure group $G$. Any such bundle is isomorphic to the fibre bundle $P \times_{G} G / H$ associated to a smooth $G$-principal 
bundle $P \rightarrow B$ and the left action of $G$ on $G / H$. Up to isomorphism a $G / H$-bundle is obtained by pulling back the universal bundle $\pi: B H \rightarrow B G$ via a map $B \rightarrow B G$ (here $\pi$ is the map of classifying spaces induced by the inclusion $H \hookrightarrow G$ ).

Let $\varphi: \Omega_{*}^{S O} \rightarrow R$ be a homomorphism to a ring $R$ with unit (recall that $\varphi$ is called a (rational) genus if $R$ is a $\mathbb{Q}$-algebra). The homomorphism $\varphi$ is multiplicative in $G / H$-bundles if $\varphi(E)=\varphi(B) \cdot \varphi(G / H)$ for any $G / H$-bundle $E \rightarrow B$. By comparing $E$ with the trivial bundle $B \times G / H$ one sees that $\varphi$ is multiplicative if and only if $\varphi$ vanishes on all $G / H$-bundles with zero-bordant base. A universal example of a multiplicative homomorphism is given by projecting $\Omega_{*}^{S O}$ to the quotient of $\Omega_{*}^{S O}$ by the ideal generated by $G / H$-bundles with zero-bordant base. If the homogeneous space $G / H$ comes with additional structure (e.g. spin structure, string structure etc.) an analogous construction can be carried out for the corresponding bordism theory (e.g. $\Omega_{*}^{\text {Spin }}, \Omega_{*}^{\text {string }}$ etc.).

A genus $\varphi: \Omega_{*}^{S O} \rightarrow R$ is multiplicative in $G / H$-bundles if and only if its associated multiplicative sequence is strictly multiplicative in the universal bundle $\pi: B H \rightarrow B G$ (cf. [7, Prop. 21.5], [32, Section 1.2], and [20, Section 4]). By definition, the latter condition means that the multiplicative sequence applied to the tangent bundle along the fibres of $\pi: B H \rightarrow B G$ maps under the pushforward ("integration along the fibres")

$$
\pi_{!}: H^{*}(B H ; R) \rightarrow H^{*-n}(B G ; R)
$$

to $H^{0}(B G ; R) \cong R$ (here $\left.n:=\operatorname{dim} G / H\right)$. This characterization allows us to make explicit computations for the universal multiplicative genus in terms of the roots of $G$ and $H$. It goes back to work of Borel and Hirzebruch $[\mathbf{7}, \S 20]$ and in the following we will refer to it as the Borel-Hirzebruch method.

The universal multiplicative genera for projective plane bundles are closely related to index theory. For example, the signature homomorphism sign $: \Omega_{*}^{S O} \rightarrow \mathbb{Z}$ gives rise to the universal multiplicative genus for $\mathbb{C} P^{2}$-bundles and the elliptic genus $\varphi_{\text {ell }}$ is a universal multiplicative genus for $\mathbb{H} P^{2}$-bundles. Before we discuss what is known for Cayley plane bundles we briefly recall the rigidity theorem for the elliptic genus $\varphi_{\text {ell }}$ (for more information on the elliptic genus we refer to [24] and $[\mathbf{2 0}])$.

In [31] Ochanine defined an elliptic genus $\varphi: \Omega_{*}^{S O} \rightarrow R$ as a genus with logarithm $g(x)$ given by an elliptic integral, i.e.

$$
g(x):=\sum_{n \geq 0} \frac{\varphi\left(\mathbb{C} P^{2 n}\right)}{2 n+1} \cdot x^{2 n+1}=\int_{0}^{x} \frac{d t}{\sqrt{R(t)}},
$$

where $R(t):=1-2 \delta t^{2}+\epsilon t^{4}, \delta, \epsilon \in R$. Examples for elliptic genera are the signature $(\delta=\epsilon=1)$ and the $\hat{A}$-genus $\left(\delta=-\frac{1}{8}, \epsilon=0\right)$.

In his groudbreaking work [40] Witten gave an interpretation of the universal elliptic genus of a manifold $M$ in terms of the "signature" of the free loop space $\mathcal{L} M$. More precisely, Witten formally applied the Lefschetz fixed point formula to a hypothetical "signature operator" on $\mathcal{L} M$ (which should exist if $M$ is spin) to obtain an invariant $\operatorname{sign}(q, \mathcal{L} M)$ of $M$ which is given by the following series of twisted signatures

$$
\operatorname{sign}(q, \mathcal{L} M):=\operatorname{sign}\left(M, \bigotimes_{n=1}^{\infty} S_{q^{n}} T M \otimes \bigotimes_{n=1}^{\infty} \Lambda_{q^{n}} T M\right) \in \mathbb{Z}[[q]]
$$


Here $\Lambda_{t}:=\sum_{i} \Lambda^{i} \cdot t^{i}$ (resp. $\left.S_{t}:=\sum_{i} S^{i} \cdot t^{i}\right)$ denotes the exterior (resp. symmetric) power operation and $\operatorname{sign}(M, E)$ denotes the index of the signature operator twisted with the complexified vector bundle $E \otimes \mathbb{C}$. Up to a constant $\operatorname{sign}(q, \mathcal{L} M)$ is the Fourier expansion of a modular form for $\Gamma_{0}(2)$. The ring $M_{*}\left(\Gamma_{0}(2)\right)$ of such modular forms is a polynomial ring with generators $\delta$ and $\epsilon$ of weight 2 and 4 , respectively (cf. $[\mathbf{2 0}$, p. 136]) and $\operatorname{sign}(q, \mathcal{L} M)$ gives rise to the universal elliptic genus

$$
\varphi_{\text {ell }}: \Omega_{*}^{S O} \rightarrow M_{*}\left(\Gamma_{0}(2)\right) \cong \mathbb{C}[\delta, \epsilon] .
$$

The main property of the elliptic genus is its rigidity.

THEOREM 4.1 (Rigidity theorem). The elliptic genus of a spin manifold is rigid under smooth compact connected Lie group actions.

The rigidity theorem was conjectured by Witten $[\mathbf{4 0}]$ and proved by Taubes [38] (see also [8, 27]).

It follows from the rigidity theorem that $\varphi_{\text {ell }}$ is rigid on the quaternionic plane $\mathbb{H} P^{2}$ and, hence, a multiplicative genus for $\mathbb{H} P^{2}$-bundles. This can also be shown directly using the Borel-Hirzebruch method. Moreover, one finds that the elliptic genus $\varphi_{\text {ell }}$ is a universal multiplicative genus for $\mathbb{H} P^{2}$-bundles.

We now return to the problem of giving a geometric description of the universal multiplicative genus for Cayley plane bundles. Recall that the Cayley plane $\mathrm{CaP}^{2}$ is the projective plane over the octonions. As a homogeneous space $\mathrm{CaP}^{2}$ can be described as the 16-dimensional 7-connected string homogeneous space $F_{4} / \operatorname{Spin}(9)$.

By the rigidity theorem the equivariant elliptic genus of $\mathrm{CaP}^{2}$ is rigid with respect to the homogeneous action of $F_{4}$. This implies that the elliptic genus is multiplicative in $C a P^{2}$-bundles. In view of Theorem 2.5 the Witten genus is multiplicative and trivial in $\mathrm{CaP}^{2}$-bundles. As pointed out to me by Rainer Jung 15 years ago both facts can be proved directly using the Borel-Hirzebruch method. For example, the vanishing of the Witten genus for $\mathrm{CaP}^{2}$-bundles is equivalent to the Jacobi triple identity for the Weierstrass' sigma function (cf. [39, p. 451]).

Jung has used the Borel-Hirzebruch method to characterize the universal multiplicative genus for $\mathrm{Ca} \mathrm{P}^{2}$-bundles in terms of a differential equation. A consequence of this description is the fact that any genus which is multiplicative in $\mathrm{CaP}^{2}$-bundles is uniquely determined by its values on $M_{4}, M_{8}, M_{12}$ and $M_{16}$, where $M_{4}$ is a $K_{3^{-}}$ surface, $M_{8}$ and $M_{12}$ are almost parallelizable manifolds of dimension 8 and 12, respectively, with non-vanishing top Pontrjagin class and $M_{16}$ is the Cayley plane $\mathrm{CaP}^{2}$ (this also follows from Proposition 2.9).

Note that the multiplicative properties of the elliptic genus and the Witten genus for $\mathrm{CaP}^{2}$-bundles are analogous to those of the signature and $\hat{A}$-genus for $\mathbb{H} P^{2}$-bundles. Taking this analogy even further we expect that the universal multiplicative genus for $\mathrm{Ca} P^{2}$-bundles will have interesting geometric properties as in the case of $\mathbb{H} P^{2}$-bundles.

In [23] Kreck and Stolz gave a description of elliptic homology based on $\mathbb{H} P^{2}$ bundles. A better understanding of the universal multiplicative genus for $\mathrm{CaP}^{2}$ bundles may be important for the construction of a generalized homology theory based on $C a P^{2}$-bundles.

Problem 4.2. Give a geometric description of the universal multiplicative genus for Cayley plane bundles. 
We find it tempting to think of the universal multiplicative genus for $\mathrm{CaP}^{2}$ bundles as a homomorphism which assigns to a string manifold $M$ the $S^{1} \times S^{1}$ equivariant "signature" of the double free loop space $\mathcal{L} \mathcal{L} M$. Unfortunately, a formal application of the Lefschetz fixed point formula to a hypothetical "signature operator" on $\mathcal{L} \mathcal{L} M$ leads to renormalization problems for infinite products in two variables which we haven't been able to resolve.

\section{A rational basis sequence related to the kernel of the Witten genus}

In this section we prove the characterization of the rational oriented bordism and the kernel of the Witten genus in terms of equivariant Cayley plane bundles given in Proposition 2.9 and Theorem 2.8. We first construct a family of $S^{3}$ equivariant Cayley plane bundles over $B S^{1}$ with non-vanishing Milnor class (see Lemma 5.1 below). These bundles will be used to exhibit a rational basis sequence with the properties given in Proposition 2.9.

For the following facts about the Cayley plane we refer to $[\mathbf{6}, \S 19]$ and the literature cited therein. The 52-dimensional compact simple sporadic Lie group $F_{4}$ has a closed subgroup isomorphic to $\operatorname{Spin}(9)$ which is unique up to inner automorphism. We fix such a subgroup and identify it with $\operatorname{Spin}(9)$. The Cayley plane $F_{4} / \operatorname{Spin}(9)$ is a 7 -connected 16-dimensional manifold with integral cohomology given by $H^{0} \cong H^{8} \cong H^{16} \cong \mathbb{Z}$ and $H^{i}=0$ otherwise.

Let $T_{S p i n}$ be the maximal torus of $\operatorname{Spin}(9) \hookrightarrow F_{4}$ which covers the standard torus $T_{S O}$ of $S O(9)$ and let $\hat{x}_{1}, \ldots, \hat{x}_{4}$ denote the standard basis of the Lie algebra of $T_{S O}$. We will use $\hat{x}_{1}, \ldots, \hat{x}_{4}$ also as a basis of the Lie algebra of $T_{S p i n}$. Thus the integral lattice of $T_{S p i n}$ is given by $a_{1} \hat{x}_{1}+\ldots+a_{4} \hat{x}_{4}$, where $a_{i} \in \mathbb{Z}$ and $a_{1}+\ldots+a_{4}$ is even. Let $x_{1}, \ldots, x_{4}$ denote the dual basis of $\hat{x}_{1}, \ldots, \hat{x}_{4}$. We will identify $x_{i}$ with an element in $H^{2}\left(B T_{S p i n} ; \mathbb{Q}\right)$ (via transgression) which is also denoted by $x_{i}$. Let $\hat{x}$ be a fixed generator of the integral lattice for $S^{1}$ and let $x$ denote the corresponding class in $H^{2}\left(B S^{1} ; \mathbb{Z}\right)$.

Let $\xi$ be the bundle $B \operatorname{Spin}(9) \rightarrow B F_{4}$ induced by the inclusion $\operatorname{Spin}(9) \hookrightarrow$ $F_{4}$. More precisely we fix a universal $F_{4}$-principal bundle with total space $E$, put $B \operatorname{Spin}(9):=E / \operatorname{Spin}(9), B F_{4}:=E / F_{4}$ and define $\xi$ by the projection map $E / \operatorname{Spin}(9) \rightarrow E / F_{4}$. The bundle $\xi$ has as fibre the Cayley plane $F_{4} / \operatorname{Spin}(9)$. Consider the monomorphism $f: S^{1} \hookrightarrow T_{\text {Spin }}$ for which the differential $d f$ maps $\hat{x}$ to $2 \hat{x}_{1}$. It is straightforward to check that the structure group of $\eta:=(B f)^{*}(\xi)$ allows a reduction to $f\left(S^{1}\right)$.

Next consider a subgroup $S^{3} \hookrightarrow F_{4}$ of the centralizer of $f\left(S^{1}\right)$ in $F_{4}$ which acts non-trivially on $F_{4} / \operatorname{Spin}(9)$ (for example choose the subgroup $S^{3} \cong \operatorname{Spin}(3) \subset$ $\operatorname{Spin}(9)$ which covers $1 \times S O(3) \subset S O(9))$. Since $S^{3}$ commutes with the structure group $f\left(S^{1}\right)$ of $\eta$ the bundle $\eta$ has a non-trivial $S^{3}$-action along the fibres.

Let $T^{\triangle} \eta$ and $T^{\triangle} \xi$ denote the tangent bundle along the fibres of $\eta$ and $\xi$, respectively (cf. $[\mathbf{6}, 7.4]$ for details). Then $T^{\triangle} \xi$ may be identified with the vector bundle $E \times_{F_{4}} T\left(F_{4} / \operatorname{Spin}(9)\right)$ (where the action of $F_{4}$ on $T\left(F_{4} / \operatorname{Spin}(9)\right)$ is induced from left multiplication) and $(B f)^{*}\left(T^{\triangle} \xi\right) \cong T^{\triangle} \eta$.

We want to show that the Milnor class $s_{2 k}\left(T^{\triangle} \eta\right)$ does not vanish for $k \geq 4$. Recall that the Milnor class $s_{2 k}(\mu)$ of a real vector bundle $\mu$ with formal Pontrjagin roots $\left\{ \pm y_{i}\right\}_{i=1, \ldots, l}$ is given by $\sum_{i=1}^{l} y_{i}^{2 k}$. As a tool we use integration over the fibres.

We will explain this in the general situation first: Let $G$ be a compact connected Lie group, $U$ a closed connected subgroup of maximal rank and $T$ a maximal torus 
of $U$. The Weyl groups of $G$ and $U$ are denoted by $W(G)$ and $W(U)$. Now choose a set $\left\{r_{i}\right\}_{i=1, \ldots, s}$ by fixing a sign for every complementary root $\pm r_{i}$ of $U \hookrightarrow G$. Let $\xi: B U \stackrel{\pi}{\rightarrow} B G$ be the induced bundle with fibre $G / U$ and $T^{\triangle} \xi$ the tangent bundle along the fibres. By a classical result of Borel [5] the rational cohomology of $B U$ and $B G$ may be identified with the invariants of $H^{*}(B T ; \mathbb{Q})$ under the actions of the Weyl groups of $U$ and $G$, respectively. Let $\pi_{!}: H^{*}(B U ; \mathbb{Q}) \rightarrow H^{*}(B G ; \mathbb{Q})$ denote the integration over the fibres of $\xi$, where $T^{\triangle} \xi$ is oriented by $e\left(T^{\triangle} \xi\right)=\prod_{i=1}^{s} r_{i}$. Then (cf. [7, §20])

$$
\pi_{!}(y)=\sum_{w \in W(G) / W(U)} w\left(\frac{y}{\prod_{i=1}^{s} r_{i}}\right) .
$$

In the next lemma this formula is used for the inclusion $\operatorname{Spin}(9) \hookrightarrow F_{4}$.

LEMMA 5.1. The coefficient $b_{k-4}$ of $x^{2 k-8}$ in $\pi_{!}\left(s_{2 k}\left(T^{\triangle} \eta\right)\right)$ does not vanish for $k \geq 4$. In particular, the Milnor class $s_{2 k}\left(T^{\triangle} \eta\right)$ is not zero for $k \geq 4$.

Proof. We fix a $W\left(F_{4}\right)$-invariant metric on the Lie algebra $\mathfrak{t}$ of $T_{\text {Spin }}$ and extend the action to $\mathfrak{t}^{*}$, such that the canonical isomorphism $\mathfrak{t} \rightarrow \mathfrak{t}^{*}, \hat{y} \mapsto\langle\hat{y}, \quad\rangle$, is $W\left(F_{4}\right)$-equivariant. The complementary roots of $\operatorname{Spin}(9)$ in $F_{4}$ are given by $\left\{ \pm r_{j}\right\}_{j=1, \ldots, 8}=\left\{\frac{1}{2}\left( \pm x_{1} \pm x_{2} \pm x_{3} \pm x_{4}\right)\right\}$ (cf. [6, §19]). Note that the Euler characteristic of the Cayley plane $F_{4} / \operatorname{Spin}(9)$ is equal to 3 . Thus, the Weyl group of $\operatorname{Spin}(9)$ has index 3 in $W\left(F_{4}\right)$. As a set of representatives of $W\left(F_{4}\right) / W(\operatorname{Spin}(9))$ we choose $\left\{1, w, w^{2}\right\}$, where

$$
w:=s_{2 x_{4}} \circ s_{\frac{1}{2}\left(x_{1}-x_{2}-x_{3}+x_{4}\right)}
$$

and $s_{\alpha}$ denotes reflection along the hyperplane $\langle\alpha, \quad\rangle=0$. More explicitly, $w$ is given by

$$
\begin{aligned}
& x_{1}+x_{2} \mapsto x_{1}+x_{2}, \quad x_{1}-x_{2} \mapsto x_{3}+x_{4}, \\
& x_{3}+x_{4} \mapsto x_{3}-x_{4}, \quad x_{3}-x_{4} \mapsto x_{1}-x_{2} .
\end{aligned}
$$

If we put

$$
y:=\frac{x_{1}+x_{2}}{2}, y_{1}:=\frac{x_{1}-x_{2}}{2}, y_{2}:=\frac{x_{3}+x_{4}}{2}, y_{3}:=\frac{x_{3}-x_{4}}{2}
$$

and define

$$
P\left(y, y_{1}, y_{2}, y_{3}\right):=\frac{\sum_{z \in\left\{y, y_{1}\right\}}\left(\left(z+y_{2}\right)^{2 k}+\left(z-y_{2}\right)^{2 k}+\left(z+y_{3}\right)^{2 k}+\left(z-y_{3}\right)^{2 k}\right)}{\left(y^{2}-y_{2}^{2}\right)\left(y^{2}-y_{3}^{2}\right)\left(y_{1}^{2}-y_{2}^{2}\right)\left(y_{1}^{2}-y_{3}^{2}\right)}
$$

then, by formula $(5.1), \pi_{!}\left(s_{2 k}\left(T^{\triangle} \xi\right)\right)$ is equal to

$$
Q\left(y, y_{1}, y_{2}, y_{3}\right):=P\left(y, y_{1}, y_{2}, y_{3}\right)+P\left(y, y_{2}, y_{3}, y_{1}\right)+P\left(y, y_{3}, y_{1}, y_{2}\right) .
$$

The coefficient of $x_{1}^{2 k-8}$ in $\pi_{!}\left(s_{2 k}\left(T^{\triangle} \xi\right)\right)$ is equal to $Q\left(\frac{1}{2}, \frac{1}{2}, 0,0\right)$. Now $P\left(\frac{1}{2}, \frac{1}{2}, 0,0\right)$ can be computed directly and is equal to

$$
2^{8-2 k} \cdot 2^{3} \text {. }
$$


If we take common denominators for $P\left(y, y_{2}, y_{3}, y_{1}\right)+P\left(y, y_{3}, y_{1}, y_{2}\right)$, put $y_{3}$ equal to zero, divide out $y_{2}^{2}$, put $y_{2}$ equal to zero, take the limit $y_{1} \rightarrow y$ and finally put $y=\frac{1}{2}$, we obtain

$$
2^{8-2 k} \cdot\left(-2^{2}-2^{2 k}+k(2 k-1)(2 k-2)\right)
$$

as value for $P\left(y, y_{2}, y_{3}, y_{1}\right)+P\left(y, y_{3}, y_{1}, y_{2}\right)$ at $\left(y, y_{1}, y_{2}, y_{3}\right)=\left(\frac{1}{2}, \frac{1}{2}, 0,0\right)$. The coefficient $c_{k-4}$ of $x_{1}^{2 k-8}$ in $\pi_{!}\left(s_{2 k}\left(T^{\triangle} \xi\right)\right)$ is equal to the sum of (5.2) and (5.3). It follows that $c_{k-4}$ is equal to $2^{8-2 k}\left(2^{2}-2^{2 k}+k(2 k-1)(2 k-2)\right)$ and is negative for $k \geq 4$. In fact, the sequence $\left\{2^{2}-2^{2 k}+k(2 k-1)(2 k-2)\right\}_{k \geq 4}$ is monotonously decreasing and starts with -84 .

Recall that $\eta$ is defined as the pullback of $\xi: B \operatorname{Spin}(9) \rightarrow B F_{4}$ with respect to $B f: B S^{1} \rightarrow B T_{\text {Spin }}$, where $d f$ maps $\hat{x}$ to $2 \hat{x}_{1}$. So if $x \in H^{2}\left(B S^{1} ; \mathbb{Z}\right)$ denotes the dual of $\hat{x}$, then $(B f)^{*}$ maps $x_{1}$ to $2 x$ and $x_{2}, x_{3}, x_{4}$ to zero. Furthermore $(B f)^{*}\left(T^{\triangle} \xi\right) \cong T^{\triangle} \eta$. For $k \geq 4$ we compute

$$
\begin{gathered}
\pi_{!}\left(s_{2 k}\left(T^{\triangle} \eta\right)\right)=\pi_{!}\left(s_{2 k}\left((B f)^{*}\left(T^{\triangle} \xi\right)\right)\right)=\pi_{!}\left((B f)^{*}\left(s_{2 k}\left(T^{\triangle} \xi\right)\right)\right)= \\
(B f)^{*}\left(\pi_{!}\left(s_{2 k}\left(T^{\triangle} \xi\right)\right)\right)=c_{k-4} \cdot(2 x)^{2 k-8} .
\end{gathered}
$$

Since $c_{k-4}$ is negative for $k \geq 4$ the coefficient $b_{k-4}=2^{2 k-8} \cdot c_{k-4}$ of $x^{2 k-8}$ is not zero. This implies that $s_{2 k}\left(T^{\triangle} \eta\right)$ does not vanish for $k \geq 4$.

Proposition 5.2 (Proposition 2.9). The oriented bordism ring has a rational basis sequence $\left\{M_{4 k}\right\}_{k \geq 1}$, i.e. $\Omega_{*}^{S O} \otimes \mathbb{Q} \cong \mathbb{Q}\left[M_{4}, M_{8}, \ldots\right]$, such that

(1) $M_{4 k}$ is a connected $4 k$-dimensional spin manifold for all $k$,

(2) $M_{4 k}$ is a string manifold for $k \geq 2$,

(3) $M_{4 k}$ is the total space of a Cayley plane bundle with structure group $S^{1}$ and non-trivial $S^{3}$-action along the fibres for $k \geq 4$.

Proof. A sequence $\left\{M_{4 k}\right\}_{k \geq 1}$ defines a rational basis sequence of $\Omega_{*}^{S O}$ if and only if the Milnor numbers $\left\langle s_{2 k}\left(T M_{4 k}\right),\left[M_{4 k}\right]\right\rangle$ of $M_{4 k}$ for $k \geq 1$ are non-zero (cf. [30, p. 194 and p. 216]). In order to construct a basis sequence with the desired properties we will first take a closer look at the characteristic classes of the bundle $T^{\triangle} \xi$ along the fibres.

Recall from [6, $\S 19]$, that the complementary roots of $\operatorname{Spin}(9) \hookrightarrow F_{4}$ are $\left\{ \pm r_{j}\right\}_{j=1, \ldots, 8}=\left\{\frac{1}{2}\left( \pm x_{1} \pm x_{2} \pm x_{3} \pm x_{4}\right)\right\}$. Since $p\left(T^{\triangle} \xi\right)=\prod_{j=1}^{8}\left(1+r_{j}^{2}\right)$ we get

$$
p_{1}\left(T^{\triangle} \xi\right)=2 \sum_{i=1}^{4} x_{i}^{2} \text {. }
$$

The fibre of $B \operatorname{Spin}(9) \rightarrow B F_{4}$ is 7 -connected. Hence, the classes $\sum_{i=1}^{4} x_{i}^{2}$ and $p_{1}\left(T^{\triangle} \xi\right)$ may be identified with elements of $H^{4}\left(B F_{4} ; \mathbb{Z}\right)$. Since $(B f)^{*}\left(x_{1}\right)=2 x$ we obtain $p_{1}\left(T^{\triangle} \eta\right)=\pi^{*}\left(8 x^{2}\right)$, where $\pi$ denotes the projection in the bundle $\eta$. Furthermore $w_{2}\left(T^{\triangle} \eta\right)^{2} \equiv p_{1}\left(T^{\triangle} \eta\right) \equiv 0 \bmod 2$, where again $w_{2}\left(T^{\triangle} \eta\right)$ and the mod 2 reduction of $p_{1}\left(T^{\triangle} \eta\right)$ are identified with elements in $H^{*}\left(B S^{1} ; \mathbb{Z} / 2 \mathbb{Z}\right)$. It follows that $w_{2}\left(T^{\triangle} \eta\right)$ is zero and the bundle $T^{\triangle} \eta$ is spin.

We now define a rational basis sequence of the stated type. Let $M_{4}$ be the $K_{3}$-surface given by the quartic $x_{0}^{4}+\ldots+x_{3}^{4}=0$ in $\mathbb{C} P^{3}$. It is well-known that $M_{4}$ is spin and has non-vanishing Milnor number (cf. for example [18]). Let $M_{8}$ and $M_{12}$ be almost parallelizable manifolds of dimension 8 and 12, respectively, with non-vanishing top Pontrjagin class. Such manifolds can be constructed via 
plumbing [29]. The manifolds $M_{8}$ and $M_{12}$ are string and have non-zero Milnor numbers.

We now proceed to define $M_{4 k}$ for $k \geq 4$. Let $M_{16}$ be the Cayley plane $F_{4} / \operatorname{Spin}(9)$. Let $V_{0}^{d}$ denote a point and $j_{0}$ the inclusion of the point into $B S^{1}$. So $M_{16} \cong j_{0}^{*}(\eta)$. For $k \geq 5$ let $V_{k-4}^{d} \hookrightarrow \mathbb{C} P^{2 k-4}$ be a complete intersection of complex dimension $2 k-8$ and of degree $d=\left(d_{1}, d_{2}, d_{3}, d_{4}\right)$, such that each $d_{i}>0$ and

$$
\sum_{i=1}^{4} d_{i}^{2}=8 a_{k-4}^{2}+2 k-3
$$

holds for some integer $a_{k-4} \neq 0$. It is a consequence of a theorem of Gauss on the representability of an integer as a sum of three squares (cf. [34, p. 45]) that we can always find such $d_{i}$ and $a_{k-4}$. In fact, one can choose $a_{k-4}>0$ and $d_{4}>0$ such that $8 a_{k-4}^{2}+2 k-3-d_{4}^{2}$ is either equal to $b$ or $4 b$, where $b$ is a natural number congruent to 3 or 6 modulo 8 , and by the theorem of Gauss $b$ is the sum of three non-zero integer squares.

Let $h \in H^{2}\left(V_{k-4}^{d} ; \mathbb{Z}\right)$ denote the pullback of the first Chern class of the dual Hopf bundle over $\mathbb{C} P^{2 k-4}$ with respect to the inclusion $V_{k-4}^{d} \hookrightarrow \mathbb{C} P^{2 k-4}$. Let $j_{k-4}: V_{k-4}^{d} \rightarrow B S^{1}$ be a map which classifies $a_{k-4} \cdot h$.

Now define $M_{4 k}$ as the pullback bundle of $\eta$ under $j_{k-4}: V_{k-4}^{d} \rightarrow B S^{1}$. Recall that $\eta$ admits a non-trivial $S^{3}$-action along the fibres. Therefore for each $k \geq 4$ the manifold $M_{4 k}$ has also a non-trivial $S^{3}$-action along the fibres as it is a pullback bundle of $\eta$.

We claim that $M_{4 k}$ is string. Since $M_{16}$ is 7 -connected $M_{16}$ is clearly string. Let $k \geq 5$. We will use the computation of the Chern classes and Pontrjagin classes of $V_{k-4}^{d}$ given in [18]. Since

$$
c_{1}\left(V_{k-4}^{d}\right)=\left(2 k-3-\sum_{i=1}^{4} d_{i}\right) h \equiv\left(2 k-3-\sum_{i=1}^{4} d_{i}^{2}\right) h \equiv-8 a_{k-4}^{2} \cdot h \equiv 0 \bmod 2
$$

the manifold $V_{k-4}^{d}$ is spin. The tangent bundle of $M_{4 k}$ splits as the direct sum of the tangent bundle along the fibres, $T^{\triangle} M_{4 k} \cong j_{k-4}^{*}\left(T^{\triangle} \eta\right)$ and the pullback of the tangent bundle of $V_{k-4}^{d}$ under the projection $\pi: M_{4 k} \rightarrow V_{k-4}^{d}$, i.e.

$$
T M_{4 k} \cong j_{k-4}^{*}\left(T^{\triangle} \eta\right) \oplus \pi^{*}\left(T V_{k-4}^{d}\right) \text {. }
$$

Since $T^{\triangle} \eta$ and $T V_{k-4}^{d}$ are spin the same holds for $M_{4 k}$. For the first Pontrjagin class of $V_{k-4}^{d}$ we obtain using equation (5.4)

$$
p_{1}\left(V_{k-4}^{d}\right)=\left((2 k-3)-\sum_{i=1}^{4} d_{i}^{2}\right) \cdot h^{2}=-8 a_{k-4}^{2} \cdot h^{2} .
$$

Since $T M_{4 k} \cong j_{k-4}^{*}\left(T^{\triangle} \eta\right) \oplus \pi^{*}\left(T V_{k-4}^{d}\right)$ we get

$$
p_{1}\left(M_{4 k}\right)=\pi^{*}\left(p_{1}\left(V_{k-4}^{d}\right)\right)+j_{k-4}^{*}\left(p_{1}\left(T^{\triangle} \eta\right)\right)=\pi^{*}\left(-8 a_{k-4}^{2} \cdot h^{2}+8 a_{k-4}^{2} \cdot h^{2}\right)=0 .
$$

Since the fibre $F_{4} / \operatorname{Spin}(9)$ is 7 -connected and $H^{4}\left(V_{k-4}^{d} ; \mathbb{Z}\right)$ is torsion free $H^{4}\left(M_{4 k} ; \mathbb{Z}\right)$ is also torsion free. So $p_{1}\left(M_{4 k}\right)=0$ implies $\frac{p_{1}}{2}\left(M_{4 k}\right)=0$ and $M_{4 k}$ is string.

We claim that $M_{4 k}$ has non-vanishing Milnor number: by Lemma 5.1 the Milnor class $s_{2 k}\left(T^{\triangle} \eta\right)$ does not vanish for $k \geq 4$. Furthermore $\pi_{!}\left(s_{2 k}\left(T^{\triangle} \eta\right)\right)=b_{k-4} \cdot x^{2 k-8}$ 


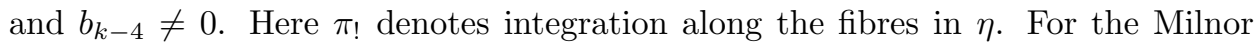
number $\left\langle s_{2 k}\left(T M_{4 k}\right),\left[M_{4 k}\right]\right\rangle$ of $M_{4 k}, k \geq 4$, we compute

$$
\begin{gathered}
\left\langle s_{2 k}\left(T M_{4 k}\right),\left[M_{4 k}\right]\right\rangle=\left\langle\pi_{!}\left(s_{2 k}\left(T M_{4 k}\right)\right),\left[V_{k-4}^{d}\right]\right\rangle= \\
\left\langle\pi_{!}\left(j_{k-4}^{*}\left(s_{2 k}\left(T^{\triangle} \eta\right)\right)+\pi^{*}\left(s_{2 k}\left(T V_{k-4}^{d}\right)\right)\right),\left[V_{k-4}^{d}\right]\right\rangle .
\end{gathered}
$$

For dimensional reasons $s_{2 k}\left(T V_{k-4}^{d}\right)$ is zero. Thus the Milnor number is equal to

$$
\left\langle j_{k-4}^{*}\left(\pi_{!}\left(s_{2 k}\left(T^{\triangle} \eta\right)\right)\right),\left[V_{k-4}^{d}\right]\right\rangle=\left\langle j_{k-4}^{*}\left(b_{k-4} \cdot x^{2 k-8}\right),\left[V_{k-4}^{d}\right]\right\rangle=b_{k-4} \cdot\left(a_{k-4} \cdot h\right)^{2 k-8} \text {. }
$$

Since $h^{2 k-8}$ is a generator of $H^{4 k-16}\left(V_{k-4}^{d} ; \mathbb{Q}\right)$ and $a_{k-4}$ and $b_{k-4}$ are non-zero the Milnor number is non-zero. Thus $\left\{M_{4 k}\right\}_{k \geq 1}$ defines a rational basis sequence for the oriented bordism ring, i.e. $\Omega_{*}^{S O} \otimes \mathbb{Q} \cong \mathbb{Q}\left[M_{4}, M_{8}, \ldots\right]$. By construction $M_{4 k}$ is a connected spin manifold for $k \geq 1$, is string for $k \geq 2$ and is a Cayley plane bundle with structure group $S^{1}$ and non-trivial $S^{3}$-action along the fibres for $k \geq 4$.

Using this proposition we can now prove Theorem 2.8 .

THEOREM 5.3 (Theorem 2.8). Let $M$ be a string manifold with $\varphi_{W}(M)=0$. Then a non-zero multiple of $M$ is string cobordant to a string manifold which is the total space of a Cayley plane bundle with structure group $S^{1}$ and non-trivial $S^{3}$-action along the fibres.

Proof. Let $M$ be a string manifold. Then any Pontrjagin number of $M$ involving $p_{1}(M)$ vanishes. Since $p_{1}\left(M_{4 k}\right)=0$ for $k \geq 2$ it follows that the rational oriented bordism class of $M$ is an element in the subring $\mathbb{Q}\left[M_{8}, M_{12}, \ldots\right]$ of $\Omega_{*}^{S O} \otimes \mathbb{Q}$.

We want to show that $M$ is in the ideal of $\mathbb{Q}\left[M_{8}, M_{12}, \ldots\right]$ which is generated by $M_{4 k}, k \geq 4$. Recall that $M_{8}$ and $M_{12}$ are almost parallelizable manifolds with nonvanishing top Pontrjagin class. The coefficients of $p_{2}$ and $p_{3}$ in the multiplicative series for the $\hat{A}$-genus are both non-zero (cf. $[\mathbf{1 9}, \S 1]$ ). So $\hat{A}\left(M_{8}\right)$ and $\hat{A}\left(M_{12}\right)$ are non-zero. Since the constant term of the $q$-power series $\varphi_{W}$ is equal to the $\hat{A}$-genus we conclude that $\varphi_{W}\left(M_{8}\right)$ and $\varphi_{W}\left(M_{12}\right)$ are non-zero.

We claim that $\varphi_{W}\left(M_{8}\right)$ and $\varphi_{W}\left(M_{12}\right)$ are algebraically independent. To see this recall that the value of the Witten genus on a $4 k$-dimensional string manifold is the $q$-expansion of a modular form of weight $2 k$ for $S L_{2}(\mathbb{Z})$. The ring $M_{*}\left(S L_{2}(\mathbb{Z})\right)$ of modular forms for $S L_{2}(\mathbb{Z})$ is a polynomial ring with one generator of weight 4 and one generator of weight 6 (cf. $\left[34\right.$, p. 89]). Since $\varphi_{W}\left(M_{8}\right)$ and $\varphi_{W}\left(M_{12}\right)$ are non-zero they can be used as generators of $M_{*}\left(S L_{2}(\mathbb{Z})\right)$. In particular, $\varphi_{W}\left(M_{8}\right)$ and $\varphi_{W}\left(M_{12}\right)$ are algebraically independent.

As $\varphi_{W}(M)=0$ we conclude from the proposition above that the rational oriented bordism class of $M$ is an element in the ideal of $\mathbb{Q}\left[M_{8}, M_{12}, \ldots\right]$ generated by $M_{4 k}, k \geq 4$. Now assume $\operatorname{dim} M \geq 16$. From the proposition above it also follows that a non-zero multiple of $M$, say $r M$, is $S O$-cobordant to a string manifold $N$ which is the total space of a Cayley plane bundle with structure group $S^{1}$ and non-trivial $S^{3}$-action along the fibres. Consider the bordism ring $\Omega_{*}^{\text {string }}$ of string manifolds. By standard arguments using the Pontrjagin-Thom construction one knows that the forgetful homomorphism

$$
\Omega_{*}^{\text {string }} \otimes \mathbb{Q} \rightarrow \Omega_{*}^{S O} \otimes \mathbb{Q}
$$

is injective. Since $r M$ and $N$ are $S O$-cobordant string manifolds $n \cdot r M$ is string cobordant to $n \cdot N$ for some non-zero integer $n$. This proves the theorem. 


\section{References}

1. Michael F. Atiyah and Friedrich Hirzebruch, Spin-manifolds and group actions., Essays Topol. Relat. Top., Mem. dediés à Georges de Rham 18-28 (1970)., 1970.

2. Michael F. Atiyah and I.M. Singer, The index of elliptic operators. III., Ann. Math. (2) 87 (1968), 546-604.

3. V.N. Berestovskij, Homogeneous Riemannian manifolds of positive Ricci curvature., Math. Notes 58 (1995), no. 3, 905-909.

4. Christoph Böhm and Burkhard Wilking, Nonnegatively curved manifolds with finite fundamental groups admit metrics with positive Ricci curvature., Geom. Funct. Anal. 17 (2007), no. 3, 665-681.

5. Armand Borel, Sur la cohomologie des espaces fibrés principaux et des espaces homogènes de groupes de Lie compacts., Ann. Math. (2) 57, 115-207.

6. Armand Borel and Friedrich Hirzebruch, Characteristic classes and homogeneous spaces. I, Am. J. Math. 80 (1958), 458-538.

7. _ Characteristic classes and homogeneous spaces. II, Am. J. Math. 81 (1959), 315-382.

8. Raoul Bott and Clifford Taubes, On the rigidity theorems of Witten., J. Am. Math. Soc. 2 (1989), no. 1, 137-186.

9. Dan Burghelea, Free differentiable $S^{1}$ and $S^{3}$ actions on homotopy spheres., Ann. Sci. École Norm. Sup. (4) 5 (1972), 183-215.

10. Anand Dessai, The Witten genus and $S^{3}$-actions., preprint (1994).

11. __ Spin ${ }^{c}$-manifolds with Pin(2)-action., Math. Ann. 315 (1999), no. 4, 511-528.

12. Characteristic numbers of positively curved spin-manifolds with symmetry., Proc. Am. Math. Soc. 133 (2005), no. 12, 3657-3661.

13. , Obstructions to positive curvature and symmetry., Adv. Math. 210 (2007), no. 2, $560-577$.

14. _ The Witten genus of string biquotients., preprint (work in progress) (2009).

15. Jost-Hinrich Eschenburg, Freie isometrische Aktionen auf kompakten Lie-Gruppen mit positiv gekrümmten Orbiträumen., Tech. report, Schriftenr. Math. Inst. Univ. Münster, II. Ser. 32, 177 S. , 1984.

16. Markus Förster, On the Stolz conjecture for generalized complete intersections in irreducible, compact, Hermitian, symmetric spaces., Münster: Univ. Münster, Fachbereich Mathematik und Informatik (Dissertation). 122 p., 2007.

17. Karsten Grove and Wolfgang Ziller, Cohomogeneity one manifolds with positive Ricci curvature., Invent. Math. 149 (2002), no. 3, 619-646.

18. Friedrich Hirzebruch, Der Satz von Riemann-Roch in Faisceau-theoretischer Formulierung; einige Anwendungen und offene Fragen. (The theorem of Riemann-Roch in Faisceau-theoretic formulation; some applications and open questions)., Proc. internat. Congr. Math. 1954 Amsterdam 3, 457-473 (1956)., 1956.

19. - Neue topologische Methoden in der algebraischen Geometrie. (New methods in algebraic geometry)., Ergebnisse der Mathematik und ihrer Grenzgebiete. 9. Berlin-GöttingenHeidelberg: Springer-Verlag. VIII, 165 S. , 1956.

20. Friedrich Hirzebruch, Thomas Berger, and Rainer Jung, Manifolds and modular forms. Transl. by Peter S. Landweber., Aspects of Mathematics. E 20. Wiesbaden: Friedr. Vieweg. xi, 211 p. , 1992.

21. Nigel J. Hitchin, Harmonic spinors., Adv. Math. 14 (1974), 1-55.

22. M.J. Hopkins, Algebraic topology and modular forms., Proc. internat. Congr. Math. 2002 Beijing I, 291-317 (2002)., 2002.

23. Matthias Kreck and Stephan Stolz, HP $\mathbf{H}^{2}$-bundles and elliptic homology., Acta Math. 171 (1993), no. 2, 231-261

24. Peter S. Landweber, Elliptic cohomology and modular forms., Elliptic curves and modular forms in algebraic topology, Proc. Conf., Princeton/NJ 1986, Lect. Notes Math. 1326 55-68 (1988)., 1988.

25. H.Blaine jun. Lawson and Shing Tung Yau, Scalar curvature, non-Abelian group actions, and the degree of symmetry of exotic spheres., Comment. Math. Helv. 49 (1974), 232-244.

26. A. Lichnerowicz, Spineurs harmoniques., C. R. Acad. Sci., Paris 257 (1963), 7-9.

27. Kefeng Liu, On modular invariance and rigidity theorems., J. Differ. Geom. 41 (1995), no. 2, 343-396. 
28. John W. Milnor, Spin structures on manifolds., Enseignement Math. (2) 9 (1963), 198-203.

29. John W. Milnor and M.A. Kervaire, Bernoulli numbers, homotopy groups, and a theorem of Rohlin., Proc. Int. Congr. Math. 1958, 454-458 (1960)., 1960.

30. John W. Milnor and James D. Stasheff, Characteristic classes., Annals of Mathematics Studies. No.76. Princeton, N.J.: Princeton University Press and University of Tokyo Press. VII, 331 p., 1974.

31. Serge Ochanine, Sur les genres multiplicatifs définis par des intégrales elliptiques. (On multiplicative genera defined by elliptic integrals)., Topology 26 (1987), 143-151.

32. __ Genres elliptiques équivariants. (Equivariant elliptic genera)., Elliptic curves and modular forms in algebraic topology, Proc. Conf., Princeton/NJ 1986, Lect. Notes Math. 1326, 107-122 (1988)., 1988.

33. Lorenz J. Schwachhöfer and Wilderich Tuschmann, Metrics of positive Ricci curvature on quotient spaces., Math. Ann. 330 (2004), no. 1, 59-91.

34. Jean-Pierre Serre, A course in arithmetic., Graduate Texts in Mathematics. 7. New YorkHeidelberg-Berlin: Springer- Verlag, 5th printing, 1996.

35. Stephan Stolz, Simply connected manifolds of positive scalar curvature., Ann. of Math. (2) 136 (1992), no. 3, 511-540.

36. A conjecture concerning positive Ricci curvature and the Witten genus., Math. Ann. 304 (1996), no. 4, 785-800.

37. Stephan Stolz and Peter Teichner, What is an elliptic object?, Tillmann, Ulrike (ed.), Topology, geometry and quantum field theory. Proceedings of the 2002 Oxford symposium in honour of the 60th birthday of Graeme Segal, Oxford, UK, June 24-29, 2002. Cambridge: Cambridge University Press. London Mathematical Society Lecture Note Series 308, 247-343 (2004)., 2004.

38. Clifford Henry Taubes, $S^{1}$-actions and elliptic genera., Commun. Math. Phys. 122 (1989), no. $3,455-526$.

39. E. T. Whittaker and G. N. Watson, A course of modern analysis. An introduction to the general theory of infinite processes and of analytic functions; with an account of the principal transcendental functions. Third edition., Cambridge: University Press, IV u. 608 S. , 1920.

40. Edward Witten, The index of the Dirac operator in loop space., Elliptic curves and modular forms in algebraic topology, Proc. Conf., Princeton/NJ 1986, Lect. Notes Math. 1326, 161-181 (1988)., 1988

41. Shing-Tung Yau, On the Ricci curvature of a compact Kähler manifold and the complex Monge-Ampère equation. I., Commun. Pure Appl. Math. 31 (1978), 339-411.

Department of Mathematics, University of Fribourg, Switzerland E-mail address: anand.dessai@unifr.ch 